\title{
On the density of truth in modal logics
}

\section{Zofia Kostrzycka}

\author{
University of Technology
}

Luboszycka 3, 45-036 Opole, Poland

E-mail zkostrzepo.opole.pl

The aim of this paper is counting the probability that a random modal formula is a tautology. We examine $\{\rightarrow, \square\}$ fragment of two modal logics S5 and S4 over the language with one propositional variable. Any modal formula written in such a language may be interpreted as a unary binary tree. As it is known, there are finitely many different formulas written in one variable in the logic S5 and this is the key to count the proportion of tautologies of S5 among all formulas. Although the logic $\mathbf{S} 4$ does not have this property, there exist its normal extensions having finitely many non-equivalent formulas.

Keywords: density of truth, Grzegorczyk's logic, S5 modal logic

\section{Introduction}

Modal logic is a widely applicable method of reasoning for many areas of computer science. These areas include artificial intelligence, database theory, distributed systems, programs verification and cryptography theory (see for example (1), (8)). Computer scientists have examined the difficulty of problems in modal logic, such as satisfiability. Satisfiability determines whether a formula in a given logic is satisfiable. Our approach also concerns the satisfiable formulas but in a quite different way. We are interested in counting the proportions of such formulas among all formulas of some given modal logic. The complexity of this problem involves we analyze some well described fragments of modal logic.

This paper consists of two parts. The first one is an algebraical characterization of two modal algebras: one-generated S5 algebra and one-generated algebra for Grzegorczyk's modal logic. We chose these modal logics because they have good semantical properties. We are especially interested in their relational semantic, which is known as Kripke frames and models. We start with analysis of the appropriate frame. Then we characterize its algebraical counterpart, which is some modal algebra (i) By the one-generated modal algebra we mean the algebra obtained from one non-zero and non-unit element by closing it under the operations. For details see (2).

The second part is devoted to calculation of numbers of formulas being tautologies of the considered logics. We are especially interested in asymptotic properties of the fractions of tautologies. Let $L$ be some logical calculus. Let $\left|T_{n}\right|$ be a number of tautologies of length $n$ of that calculus and $\left|F_{n}\right|$ be a number of all formulas of that length. We define the density $\mu(L)$ as:

$$
\mu(L)=\lim _{n \rightarrow \infty} \frac{\left|T_{n}\right|}{\left|F_{n}\right|}
$$

If the limit exists, then it is the probability that a random formula is a tautology and is called density of truth. This paper is continuation of research concerning the density of truth in different logics. Until now, the density of truth for classical (and intuitionistic) logic of implication of one and two variables are known (see (15),(9)) as well as the density of implicational-negational fragments of that calculus with one variable (see (18),(13)). There are also many results concerning the density of truth of classical logic with the connectives of conjunction, disjunction and negation. For example, see (3) and (6). Regarding modal logics, there is made only a rough estimation of the density of truth for Grzegorczyk's logic. It is proved in (11) that

$$
34.672 \%<\mu(\mathbf{G r z})<61.27 \%
$$

In this paper we significantly improve the above estimation and compare this result with the one for the logic S5. The methods described in (3), (6) and (5) will be used.

(i) A structure $\mathfrak{A}=\langle A, \cap, \cup,-, I, 0,1\rangle$ is a modal algebra if: (1) $\langle A, \cap, \cup,-, 0,1\rangle$ is a Boolean algebra, and (2) $I$ is a unary operator that satisfies (i) $I(1)=1$ and (ii) $I(a \cap b) I(a) \cap I(b)$ for $a, b \in A$. 


\section{Normal extensions of S4 modal logic. Modal logic S5}

Syntactically, the modal logic $\mathbf{S} 4$ is obtained by adding to axioms of classical logic the following modal formulas ${ }^{\text {(ii) }}$ (re $) \square p \rightarrow p,(K) \square(p \rightarrow q) \rightarrow(\square p \rightarrow \square q)$, (tra) $\square p \rightarrow \square \square p$. The logic $\mathbf{S} 4$ is defined as a set of all consequences of new axioms by modus ponens, substitution and necessitation $\left(R_{G}\right)$ rules. The last one can be presented in the following scheme: $\vdash \alpha / \vdash \square \alpha$.

By a frame we mean a pair $\mathfrak{F}=\langle W, R\rangle$ consisting of a nonempty set $W$ and a binary relation $R$ on $W$. The elements of $W$ are called points (or worlds) and $x R y$ is read as ' $y$ is accessible from $x$ '. A modal proposition $\square \varphi$ is regarded to be true in a world $x$ if $\varphi$ is true in all the worlds alternative (being in relation $R$ ) to $x$; $\diamond \varphi$ is true in $x$ if $\varphi$ is true at least in one alternative world. Concrete properties of the alternativeness relation depends on the type of the modality under consideration. For the $\mathbf{S} 4$ logic the relation $R$ should be reflexive and transitive. By $x \uparrow$ we mean the set of successors of $x$ and by $x \downarrow$-the set of its predecessors.

The Grzegorczyk logic Grz is characterized as a normal extension of the Lewis calculus $\mathbf{S} 4$ by the Grzegorczyk axiom: $($ grz $) \square(\square(p \rightarrow \square p) \rightarrow p) \rightarrow p$.

Symbolically, the normal extension is written by $\mathbf{G r z}=\mathbf{S} 4 \oplus(\mathbf{g r z})$, where $\oplus$ means the closure under modus ponens, substitution and $R_{G}$.

Semantically, the Grz logic is characterized by the class of finite reflexive and transitive trees.

Recall, that by a tree we mean a rooted frame $\mathfrak{F}=\langle W, R\rangle$ such that for every point $x \in W$, the set $x \downarrow$ is finite and linearly ordered by $R$. A frame $\mathfrak{F}=\langle W, R\rangle$ is a rooted one if there is a world $x \in W$ such that for any $y \in W, y \quad x, y \in x \uparrow$. A transitive frame is rooted if there exists a point $x \in W$ such that for any $y \in W, y \in x \uparrow$.

In this section we examine normal extensions of Grzegorczyk's logic obtained by adding to the set of axioms new formulas. The considered in this section axiomatic extensions are uniformly connected with depth of trees.

Definition 1 A frame $\mathfrak{F}$ is of depth $n<\omega$ if there is a chain of $n$ points in $\mathfrak{F}$ and no chain of more than $n$ points exists in $\mathfrak{F}$.

For $n>0$, let $J_{n}$ be an axiom saying that any strictly ascending partial-ordered sequence of points is of length $n$ at most. The form of the needed formulas $J_{n}$ is well known (see (2) p.42). They are defined inductively as follows (iii)

\section{Definition 2}

$$
\begin{aligned}
J_{1} & =\diamond \square p_{1} \rightarrow p_{1}, \\
J_{n+1} & =\diamond\left(\square p_{n+1} \wedge \sim J_{n}\right) \rightarrow p_{n+1} .
\end{aligned}
$$

We will consider the logics $\mathbf{G r z}{ }^{\leq n}=\mathbf{G r z} \oplus J_{n}$. Let us notice that for any formula $\varphi$ if $\varphi \in \mathbf{G r z}^{\leq n}$ (that means $\varphi$ is true in every world of the tree of depth $n$ ), then $\varphi \in \mathbf{G r z}^{\leq n-1}$ ( $\varphi$ is true in every world of the tree of depth $n-1$ ). Hence the following inclusions hold:

$$
\operatorname{Grz} \subset \ldots \subset \mathbf{G r z}^{\leq n} \subset \mathbf{G r z}^{\leq n-1} \subset \ldots \subset \mathbf{G r z}^{\leq 2} \subset \mathbf{G r z}^{\leq 1} .
$$

The Lewis modal logic S5 is characterized as a normal extension of $\mathbf{S} 4$ by the axiom:

(sym) $p \rightarrow \diamond \square p$.

Semantically, $\mathbf{S} \mathbf{5}$ is characterized by the class of reflexive symmetric and transitive frames. Such relation is an equivalence relation and in that case any two worlds are alternatives to each other.

Because the logics S5 and Grz are axiomatic extensions of S4 logic then the following inclusions take places: S4 $\subset$ S5 and S4 $\subset$ Grz.

The logics $\mathbf{S} 5$ and $\mathbf{G r z}$ are however incomparable. The formula $(g r z)$ is not provable in $\mathbf{S} 5$ whereas the formula $($ sym) is not a theorem of Grz.

(ii) S4 Lewis modal logic is a super-system of the logics S1, S2 and S3 and a sub-system of S5. They were first defined in 1932 in Lewis and Langford's book Symbolic Logic and to axiomatize them, the authors used the functor of so-called strict implication. For details see (7).

(iii) The formulas $J_{n}$ are defined in the full language (with negation and the operator of possibility and with unrestricted number of variables). In the language $F^{\{\rightarrow, \square\}}$ we can find the analogous formulas. In $(10)$ the author considered the sequence of following formulas defined inductively: $A_{1}:=p, A_{2 n}:=\square A_{2 n-1}, A_{2 n+1}:=A_{2 n-1} \rightarrow A_{2 n}$ for $n \geq 1$. It is shown that the formula $A_{2 n+1}$ plays the same role as the formula $J_{n}$. 


\section{One-generated fragments of $\mathrm{Grz}^{\leq 3}$ and S5}

We will consider set $F^{\{\rightarrow, \square\}}$ of formulas built up from one propositional variable $p$ by means of necessitation and implication only.

$$
\begin{aligned}
& p \in F^{\{\rightarrow, \square\}}, \\
& \alpha \rightarrow \beta \in F^{\{\rightarrow, \square\}} \quad \text { iff } \alpha \in F^{\{\rightarrow, \square\}} \text { and } \beta \in F^{\{\rightarrow, \square\}}, \\
& \square \alpha \in F^{\{\rightarrow, \square\}} \text { iff } \alpha \in F^{\{\rightarrow, \square\}} .
\end{aligned}
$$

By a one-generated logic we mean the logic restricted to formulas built up from one variable. The one-generated parts of logics $\mathbf{G r z}^{\leq 3}$ and S5, (over the language with $\rightarrow$ and $\square$ ) will be represented appropriately by the symbols $\mathbf{G r z}{ }^{\leq 3}(1)$ and $\mathbf{S 5}(1)$.

The simplest manner to characterize the logics $\mathbf{G r z}{ }^{\leq 3}(1)$ and $\mathbf{S 5}(1)$ is examining the appropriate TarskiLindenbaum algebras $\mathbf{G r z} \mathbf{z}^{\leq 3}(1) / \equiv$ and $\mathbf{S 5}(1) / \equiv$. Let us introduce an equivalence relation on the considered logics:

Definition $3 \alpha \equiv \beta$ iff $\alpha \rightarrow \beta \in \mathbf{M}$ and $\beta \rightarrow \alpha \in \mathbf{M}$, where the symbol $\mathbf{M}$ stands for $\mathbf{G r z} \leq 3(1)$ or S5(1).

In (10) the author gave a precise characterization of the quotient algebras $\operatorname{Grz}^{\leq n}(1) / \equiv$ for any $n \geq 1$. For $n=3$ as well as for $\mathbf{S 5}(1) / \equiv$ we have:

Lemma 4 Both the algebras $\mathbf{G r z} \leq^{\leq 3}(1) / \equiv$ and $\mathbf{S 5}(1) / \equiv$ consist of the following eight equivalence classes:

$$
\begin{aligned}
& A_{1}=c[p]_{\equiv} \\
& A_{3}=[p \rightarrow \square p]_{\equiv} \\
& A_{5}=A_{3} \rightarrow A_{4} \\
& B_{2}=A_{5} \rightarrow A_{2}
\end{aligned}
$$

$$
\begin{array}{cccc}
A_{2} & = & & {[\square p]_{\equiv}} \\
A_{4} & = & & {[\square(p \rightarrow \square p)]_{\equiv}} \\
B_{1} & = & A_{4} \rightarrow A_{2} \\
T & = & & {[p \rightarrow p]_{\equiv}}
\end{array}
$$

and are closed under the operations $\rightarrow$ and $\square$.

Proof. For $\mathbf{G r z}^{\leq 3}(1) / \equiv$ see $(10)$. The proof for S5 may be made by a mutual calculation or by taking the advantage of canonical and universal frames. The universal frame $\gamma_{\mathfrak{F}_{S 5}}(1)$ generated by one variable consists of one two-point cluster and one reflexive point falsifying $p$. Hence the dual algebra (which is in fact

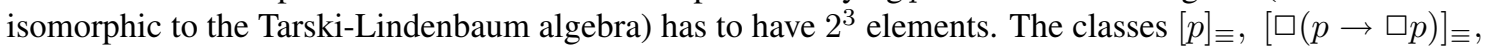
and $[((p \rightarrow \square p) \rightarrow \square(p \rightarrow \square p)) \rightarrow \square p]_{\equiv}$ are atoms of $\mathbf{S 5}(1) / \equiv$. For details we refer the reader to (2).

Let us notice, that the equivalence classes presented above, are not identical in the both cases of S5(1) and $\mathbf{G r z}^{\leq 3}(1)$ logics. They have only the same representant. To avoid misunderstanding, we propose to mark the class $T$ of tautologies of $\mathbf{G r z}{ }^{\leq 3}(1)$ by $T_{G r z}$ and analogously for $\mathbf{S 5}(1)-T_{S 5}$.

Observation 5 The operations $\{\rightarrow, \square\}$ in the algebra $\mathbf{G r z}{ }^{\leq 3}(1) / \equiv$ can be displayed in Table 1

\begin{tabular}{|c|cccccccc|c|}
\hline$\rightarrow$ & $A_{1}$ & $A_{2}$ & $A_{3}$ & $A_{4}$ & $A_{5}$ & $B_{1}$ & $B_{2}$ & $T_{G r z}$ & $\square$ \\
\hline$A_{1}$ & $T_{G r z}$ & $A_{3}$ & $A_{3}$ & $A_{3}$ & $T_{G r z}$ & $T_{G r z}$ & $A_{3}$ & $T_{G r z}$ & $A_{2}$ \\
$A_{2}$ & $T_{G r z}$ & $T_{G r z}$ & $T_{G r z}$ & $T_{G r z}$ & $T_{G r z}$ & $T_{G r z}$ & $T_{G r z}$ & $T_{G r z}$ & $A_{2}$ \\
$A_{3}$ & $A_{1}$ & $A_{1}$ & $T_{G r z}$ & $A_{5}$ & $A_{5}$ & $B_{1}$ & $B_{1}$ & $T_{G r z}$ & $A_{4}$ \\
$A_{4}$ & $B_{1}$ & $B_{1}$ & $T_{G r z}$ & $T_{G r z}$ & $T_{G r z}$ & $B_{1}$ & $B_{1}$ & $T_{G r z}$ & $A_{4}$ \\
$A_{5}$ & $B_{1}$ & $B_{2}$ & $A_{3}$ & $A_{3}$ & $T_{G r z}$ & $B_{1}$ & $B_{2}$ & $T_{G r z}$ & $A_{5}$ \\
$B_{1}$ & $A_{5}$ & $A_{4}$ & $A_{3}$ & $A_{4}$ & $A_{5}$ & $T_{G r z}$ & $A_{3}$ & $T_{G r z}$ & $A_{2}$ \\
$B_{2}$ & $A_{5}$ & $A_{5}$ & $T_{G r z}$ & $A_{5}$ & $A_{5}$ & $T_{G r z}$ & $T_{G r z}$ & $T_{G r z}$ & $A_{2}$ \\
$T_{G r z}$ & $A_{1}$ & $A_{2}$ & $A_{3}$ & $A_{4}$ & $A_{5}$ & $B_{1}$ & $B_{2}$ & $T_{G r z}$ & $T_{G r z}$ \\
\hline
\end{tabular}

Tab. 1: Truth table for $\mathbf{G r z} \leq 3(1) / \equiv$

Observation 6 The operations $\{\rightarrow, \square\}$ in the algebra $\mathbf{S 5}(1) / \equiv$ can be displayed in Table 2.

The order forms the following lattice diagrams of the eight-element $\mathbf{G r} \mathbf{z}^{\leq 3}(1) / \equiv$ and $\mathbf{S 5}(1) / \equiv$ modal algebras, which are, in fact, eight-element Boolean cubes (see Figure 1). The open circle stands for the open element of the given algebra. An element $A$ is open if $\square A=A$. Let us stress the main difference 


\begin{tabular}{|c|cccccccc|c|}
\hline$\rightarrow$ & $A_{1}$ & $A_{2}$ & $A_{3}$ & $A_{4}$ & $A_{5}$ & $B_{1}$ & $B_{2}$ & $T_{S 5}$ & $\square$ \\
\hline$A_{1}$ & $T_{S 5}$ & $A_{3}$ & $A_{3}$ & $A_{3}$ & $T_{S 5}$ & $T_{S 5}$ & $A_{3}$ & $T_{S 5}$ & $A_{2}$ \\
$A_{2}$ & $T_{S 5}$ & $T_{S 5}$ & $T_{S 5}$ & $T_{S 5}$ & $T_{S 5}$ & $T_{S 5}$ & $T_{S 5}$ & $T_{S 5}$ & $A_{2}$ \\
$A_{3}$ & $A_{1}$ & $A_{1}$ & $T_{S 5}$ & $A_{5}$ & $A_{5}$ & $B_{1}$ & $B_{1}$ & $T_{S 5}$ & $A_{4}$ \\
$A_{4}$ & $B_{1}$ & $B_{1}$ & $T_{S 5}$ & $T_{S 5}$ & $T_{S 5}$ & $B_{1}$ & $B_{1}$ & $T_{S 5}$ & $A_{4}$ \\
$A_{5}$ & $B_{1}$ & $B_{2}$ & $A_{3}$ & $A_{3}$ & $T_{S 5}$ & $B_{1}$ & $B_{2}$ & $T_{S 5}$ & $A_{4}$ \\
$B_{1}$ & $A_{5}$ & $A_{4}$ & $A_{3}$ & $A_{4}$ & $A_{5}$ & $T_{S 5}$ & $A_{3}$ & $T_{S 5}$ & $A_{2}$ \\
$B_{2}$ & $A_{5}$ & $A_{5}$ & $T_{S 5}$ & $A_{5}$ & $A_{5}$ & $T_{S 5}$ & $T_{S 5}$ & $T_{S 5}$ & $A_{2}$ \\
$T_{S 5}$ & $A_{1}$ & $A_{2}$ & $A_{3}$ & $A_{4}$ & $A_{5}$ & $B_{1}$ & $B_{2}$ & $T_{S 5}$ & $T_{S 5}$ \\
\hline
\end{tabular}

Tab. 2: Truth table for S5(1)/ $\equiv$
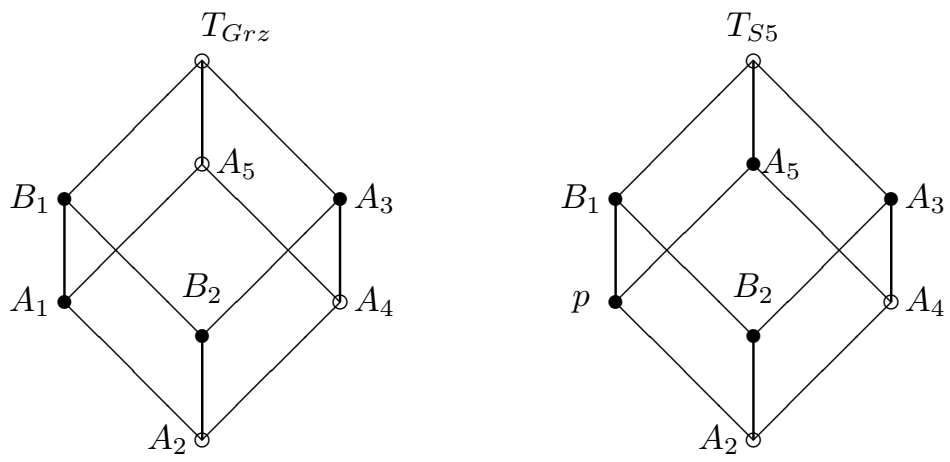

Fig. 1: Diagrams of $\mathbf{G r z}{ }^{\leq 3}(1) / \equiv$ and $\mathbf{S 5}(1) / \equiv$.

between the investigated algebras: the class $A_{5}$ is open in the algebra $\mathbf{G r z} \mathbf{z}^{\leq 3}(1) / \equiv$ whereas in $\mathbf{S 5}(1) / \equiv$ is not.

To give a satisfying characterization of the algebra $\mathrm{Grz}^{\leq 3}(1) / \equiv$ it is necessary to notice that it has two non-trivial open filters $\left[A_{5}\right)$ and $\left[A_{4}\right)$. They determine two congruence relations on $\mathbf{G r z}^{\leq 3}(1) / \equiv$. We divide the considered algebra by them and in this manner obtain some identification of classes. Exactly, we obtain the following two new quotient algebras $A L_{1}$ and $A L_{2}$.

\section{Definition 7}

$$
\begin{aligned}
& A L_{1}=\mathbf{G r z}^{\leq 3}(1) / \equiv /\left[A_{5}\right) \\
& A L_{2}=\mathbf{G r z}^{\leq 3}(1) / \equiv /\left[A_{4}\right)
\end{aligned}
$$

Their diagrams are presented in Figure 2
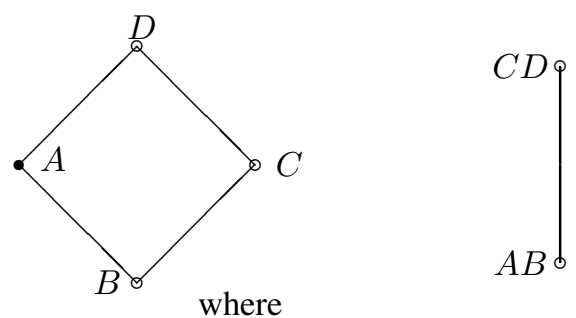

$$
\begin{array}{ll}
A=A_{1} \cup B_{1}, & B=A_{2} \cup B_{2}, \\
C=A_{3} \cup A_{4}, & D=A_{5} \cup T_{G r z}, \\
A B=A \cup B, & C D=C \cup D
\end{array}
$$

Fig. 2: Diagram of $A L_{1}$ and $A L_{2}$

There is some simple observation from (10): 


\section{Observation 8}

$$
\begin{aligned}
& A L_{1}=\mathbf{G r z}^{\leq 2}(1) / \equiv \\
& A L_{2}=\mathbf{G r z}^{\leq 1}(1) / \equiv
\end{aligned}
$$

In the algebra $\mathbf{S 5}(1) / \equiv$ there is only one open filter $\left[A_{4}\right)$. The new quotient algebra $A L_{3}=\mathbf{S 5}(1) / \equiv /\left[A_{4}\right)$ is identical with $A L_{2}$.

\section{Counting formulas and generating functions}

In this section we set up the way of counting formulas with the established length. We will consider the set $F_{n} \subseteq F^{\{\rightarrow, \square\}}$ of all formulas of length $n$.

Definition $9 B y|\phi|$ we mean the length of formula $\phi$ which is the total number of occurrences of propositional variable $p$ in the formula, including the implication and the sign of necessity operator. Formally

$$
\begin{aligned}
|p| & =1, \\
|\phi \rightarrow \psi| & =|\phi|+|\psi|+1, \\
|\square \phi| & =|\phi|+1 .
\end{aligned}
$$

Definition 10 By $F_{n}$ we mean the set of formulas from $F^{\{\rightarrow, \square\}}$ of length $n$.

We will also consider some appropriate subclasses of $F_{n}$. For example if we have a class $A \in F^{\{\rightarrow, \square\}}$ then $A_{n}=F_{n} \cap A$.

Definition $11 B y\left|A_{n}\right|$ we mean the number of formulas from the class $A_{n}$.

The main tool for dealing with asymptotics of sequences of numbers are generating functions. A nice exposition of this method can be found in (16) and (5).

Lemma 12 The generating function $f_{F}$ for the numbers $\left|F_{n}\right|$ is

$$
f_{F}(z)=\frac{1-z}{2 z}-\frac{\sqrt{(z+1)(1-3 z)}}{2 z} .
$$

Proof. It is easy to observe that the generating function $f_{F}$ for the numbers $\left|F_{n}\right|$ is the one for unary binary trees (see (5), p.65).

Suppose, we have a system of non-linear equations $\overrightarrow{y_{j}}=\Phi_{j}\left(z, y_{1}, \ldots y_{m}\right)$ for $1 \leq j \leq m$, where any $y_{j}=\sum_{n=0}^{\infty} a_{j} z^{n}$. The following result known as Drmota-Lalley-Woods theorem (see (5), Thm. 8.13, p.71) is of great importance in the both cases of solving the system explicitly or implicitly.

Theorem 13 Consider a nonlinear polynomial system, defined by a set of equations

$$
\left\{\vec{y}=\Phi_{j}\left(z, y_{1}, \ldots, y_{m}\right)\right\}, \quad 1 \leq j \leq m
$$

satisfying the following properties:

1. a-properness: $\Phi$ is a contraction, i.e. satisfies the Lipschitz condition

$$
d\left(\Phi\left(y_{1}, \ldots, y_{m}\right), \Phi\left(y_{1}^{\prime}, \ldots, y_{m}^{\prime}\right)\right)<K d\left(\left(y_{1}, \ldots, y_{m}\right),\left(y_{1}^{\prime}, \ldots, y_{m}^{\prime}\right)\right), \quad K<1 .
$$

2. a-positivity: all terms of the series $\Phi_{j}(\vec{y})$ are $\geq 0$.

3. a-irreducibility: the dependency graph of the algebraic system is built on $m$ vertices: $1,2, \ldots, m$; there is an edge from a vertex $k$ to a vertex $j$ if $y_{j}$ appears in $\phi_{k}$. The algebraic system is an irreducible if its dependency graph is strongly connected.

4. a-aperiodicity: $z$ (not $z^{2}$ or $z^{3} \ldots$ ) is the right variable, that means for each $\phi_{j}$ there exist three monomials $z^{a}, z^{b}$, and $z^{c}$ such that $b-a$ and $c-a$ are relatively prime. 
1. All component solutions $y_{i}$ have the same radius of convergence $\rho<\infty$.

2. There exist functions $h_{j}$ analytic at the origin such that

$$
y_{j}=h_{j}(\sqrt{1-z / \rho}), \quad\left(z \rightarrow \rho^{-}\right) .
$$

3. All other dominant singularities are of the form $\rho \omega$ with $\omega$ being a root of unity.

4. If the system is a-aperiodic then all $y_{j}$ have $\rho$ as unique dominant singularity. In that case, the coefficients admit a complete asymptotic expansion of the form:

$$
\left[z^{n}\right] y_{j}(z) \sim \rho^{-n}\left(\sum_{k \geq 1} d_{k} n^{-1-k / 2}\right) .
$$

From (5) there is a simple transition by the so called transfer lemma from (4), to a formula defining the value of the coefficients $\left[z^{n}\right] y_{j}(z)$. So, the a-aperiodicity of a system of equations is a very desirable property. The application of the above theorem will proceed in the following way. Suppose, we have two functions $f_{T}$ and $f_{F}$ enumerating tautologies and all formulas of some logic. Suppose, they have the same dominant singularity $\rho$ and there are the suitable constants $\alpha_{1}, \alpha_{2}, \beta_{1}, \beta_{2}$ such that:

$$
\begin{aligned}
& f_{T}(z)=\alpha_{1}-\beta_{1} \sqrt{1-z / \rho}+O(1-z / \rho), \\
& f_{F}(z)=\alpha_{2}-\beta_{2} \sqrt{1-z / \rho}+O(1-z / \rho) .
\end{aligned}
$$

Then the density of truth (probability that a random formula is a tautology) is given by:

$$
\mu(T)=\lim _{n \rightarrow \infty} \frac{\left[z^{n}\right] f_{T}(z)}{\left[z^{n}\right] f_{F}(z)} \frac{\beta_{1}}{\beta_{2}} .
$$

Let us notice that the limit (8) exists in the case of a-aperiodic system of equations. The lack of this property involves the limit does not exist (see for example (17)). Such situation is considered in (14) and in (12). In the last one the author investigate the existence of density of the purely equivalential fragments of classical and intuitionistic logics.

\section{Counting the density of $\mathrm{Grz}^{\leq 3}$}

At the begining of this section we quote (without a proof) the main generating functions for the classes of formulas from the algebras $A L_{1}$ and $A L_{2}$. From (11) we have (iv),

Lemma 14 The generating functions $f_{A B}$ and $f_{C D}$ for the numbers $\left|A B_{n}\right|$ and $\left|C D_{n}\right|$ are

$$
\begin{aligned}
f_{A B}(z) & =\frac{z f(z)-1+z+X}{2 z}, \\
f_{C D}(z) & =\frac{z f(z)+1-z-X}{2 z}, \\
X & =\sqrt{4 z^{2}+(1-z-f(z))^{2}} .
\end{aligned}
$$

For simplicity we have written this function in the term of function $f$. We will repeat it to the other one.

Proof. See the proof of Lemma 8 from (11).

\footnotetext{
(iv) There is a small change in the present approach (actually, we have changed the way of counting length of formula), hence there are some differences between the functions presented above and those ones from (11). The method of determining them is exactly the same.
} 
Lemma 15 The generating function $f_{C}$ for the numbers $\left|C_{n}\right|$ is

$$
\begin{aligned}
& f_{C}(z)=\frac{T}{4 z}+\frac{1}{2} \sqrt{X+\frac{1}{2} \sqrt{-\frac{Y}{92^{1 / 3} z^{4}}+\frac{T^{2}+4 U}{3 z^{2}}-\frac{S}{Y}+\frac{T^{3}+4 T U+R}{4 X z^{3}}}} \\
& \text { where } \\
& Y=3 z \sqrt[3]{W+\sqrt{-4\left(S z^{2}\right)^{3}+W^{2}}} \\
& W=z^{3}\left(-2 U^{3}+\frac{9}{8} T U+\frac{27}{64} R^{2}\right)-z^{7}\left(T^{2} f_{A B}+72 U f_{A B}\right), \\
& R=8\left(-1+\left(1+f-f_{A B}\right) z+\left(1+(-1+f) f_{A B}\right) z^{2}+\left(1+2(1+f) f_{A B}\right) z^{3}\right), \\
& X=\frac{Y}{92^{1 / 3} z^{4}}+\frac{T^{2}}{4 z^{2}}+\frac{2 U}{3 z^{2}}+\frac{S}{Y}, \\
& S=U^{2}-12 f_{A B} z^{4}-\frac{3}{8} T R \\
& T=-3+z+3 z f-2 z f_{A B}, \\
& U=-3+\left(2+3 f-3 f_{A B}\right) z+\left(2+f+3 f f_{A B}\right) z^{2} .
\end{aligned}
$$

For simplicity we have omitted in the above function the argument $(z)$ and have written $f$ and $f_{A B}$ instead of $f(z)$ and $f_{A B}(z)$.

Proof. See the proof of Lemma 10 from (11).

As we see the generating functions for the class $C_{n}$ is really complicated (to determine it we have to solve a four-degree equation). Because it is not essential for the paper we do not quote here the functions $f_{A} f_{B}$ and $f_{D}$. For the whole algebra $\mathbf{G r z}^{\leq 3}(1) / \equiv$ we would have to solve equation of eight degree. From Table 1 we obtain the system of the following equations:

$$
\begin{aligned}
f_{1}= & \left(f_{8} f_{1}+f_{3}\left(f_{1}+f_{2}\right)\right) z+z, \\
f_{2}= & \left(f_{8} f_{2}+f_{1}+f_{2}+f_{6}+f_{7}\right) z, \\
f_{3}= & \left(f_{1}\left(f_{2}+f_{3}+f_{4}+f_{7}\right)+f_{5}\left(f_{3}+f_{4}\right)+f_{6}\left(f_{3}+f_{7}\right)+f_{8} f_{3}\right) z, \\
f_{4}= & \left(f_{6}\left(f_{2}+f_{4}\right)+f_{8} f_{4}+f_{3}+f_{4}\right) z, \\
f_{5}= & \left(f_{3}\left(f_{4}+f_{5}\right)+f_{6}\left(f_{1}+f_{5}\right)+f_{7}\left(f_{1}+f_{2}+f_{4}+f_{5}\right)+f_{8} f_{5}+f_{5}\right) z, \\
f_{6}= & \left(f_{3}\left(f_{6}+f_{7}\right)+f_{4}\left(f_{1}+f_{2}+f_{6}+f_{7}\right)+f_{5}\left(f_{1}+f_{5}\right)+f_{8} f_{6}\right) z, \\
f_{7}= & \left(f_{5}\left(f_{2}+f_{7}\right)+f_{8} f_{7}\right) z, \\
f_{8}= & \left(f_{1}\left(f_{1}+f_{5}+f_{6}\right)+f_{2}\left(f_{1}+f_{2}+f_{3}+f_{4}+f_{5}+f_{6}+f_{7}\right)+f_{3}^{2}+\right. \\
& \left.+f_{4}\left(f_{3}+f_{4}+f_{5}\right)+f_{5}^{2}+f_{6}^{2} f_{7}\left(f_{3}+f_{6}+f_{7}\right)+f_{8} f_{F}+f_{8}\right) z,
\end{aligned}
$$

where

$$
\begin{array}{llll}
f_{1}=f_{A_{1}}, & f_{2}=f_{A_{2}}, & f_{3}=f_{A_{3}}, & f_{4}=f_{A_{4}}, \\
f_{5}=f_{A_{5}}, & f_{6}=f_{B_{1}}, & f_{7}=f_{B_{2}}, & f_{8}=f_{T_{G r z}} .
\end{array}
$$

Furthermore, we have that:

$$
\begin{aligned}
f_{F} & =f_{1}+f_{2}+f_{3}+f_{4}+f_{5}+f_{6}+f_{7}+f_{8}, \\
f_{2}+f_{7} & =f_{B} \\
f_{1}+f_{6} & =f_{A} \\
f_{3}+f_{4} & =f_{C}
\end{aligned}
$$

Application of (19)-22) into (11)-(18) gives us a simpler system:

$$
\begin{aligned}
& f_{1}=\left(f_{8} f_{1}+f_{3}\left(f_{1}+f_{2}\right)\right) z+z, \\
& f_{2}=\left(f_{8} f_{2}+f_{A B}\right) z \\
& f_{3}=\left(f_{1}\left(f_{2}+f_{C}+f_{7}\right)+f_{5} f_{C}+f_{6}\left(f_{3}+f_{7}\right)+f_{8} f_{3}\right) z, \\
& f_{4}=\left(f_{6}\left(f_{2}+f_{4}\right)+f_{8} f_{4}+f_{C}\right) z,
\end{aligned}
$$




$$
\begin{aligned}
f_{5}= & \left(f_{3}\left(f_{4}+f_{5}\right)+f_{6}\left(f_{1}+f_{5}\right)+f_{7}\left(f_{1}+f_{2}+f_{4}+f_{5}\right)+f_{8} f_{5}+f_{5}\right) z, \\
f_{6}= & \left(f_{3}\left(f_{6}+f_{7}\right)+f_{4} f_{A B}+f_{5}\left(f_{1}+f_{5}\right)+f_{8} f_{6}\right) z, \\
f_{7}= & \left(f_{5} f_{B}+f_{8} f_{7}\right) z \\
f_{8}= & \left(f_{1}\left(f_{A}+f_{5}\right)+f_{2}\left(f_{F}-f_{8}\right)+f_{3}^{2}+\right. \\
& \left.+f_{4}\left(f_{C}+f_{5}\right)+f_{5}^{2}+f_{6}^{2} f_{7}\left(f_{3}+f_{6}+f_{7}\right)+f_{8} f_{F}+f_{8}\right) z .
\end{aligned}
$$

Of course, there is possible further simplification of the above system of equations. Anyway, this also leads to 8-degree equation with very complex functions. Even with taking the advantage of the Mathematica package the needed calculations are not feasible. Hence, we decide to solve the system numerically.

Let us notice that the system of equations $(11)$ - $(18)$ is a-proper, a-positive, a-irreducible and a-aperiodic. So, it is possible to apply the Drmota-Lalley-Woods Theorem 13 Furthermore, for any $i=1, \ldots, 8$ all the functions $f_{i}$ have the same dominant singularity $z_{0}=\frac{1}{3}$ and there exist their expansions around $z_{0}$ in the form:

$$
f_{i}=a_{i}+b_{i} \sqrt{1-3 z}+O(1-3 z) .
$$

From equation (3) defining the main generating function, after a simple calculation we obtain:

\section{Lemma 16}

$$
f_{F}(z)=1-\sqrt{3} \sqrt{1-3 z}+O(1-3 z) .
$$

From the system of equations 11 - -18 , we are able to count the floating values of functions $f_{i}$ at $z_{0}=\frac{1}{3}$ for any $i=1, \ldots, 8$. We may also take the differential of each equation and solve it with respect to the value of $f_{i}^{\prime}$ at $z_{0}=\frac{1}{3}$ (v) After numerical computation (20.000 steps) we obtain the following values (rounded to five digits) for the most interesting for us function $f_{8}=f_{T_{G r z}}$.

\section{Lemma 17}

$$
f_{T_{G r z}}(z) \approx 0.31099-1.05449 \sqrt{1-3 z}+O(1-3 z) .
$$

From the above it follows that

Theorem 18 The density of truth of the logic $\mathbf{G r z}^{\leq 3}(1)$ is about $60.88 \%$.

Proof. From Lemma 16 and 17 we have:

$$
\mu\left(\mathbf{G r z}^{\leq 3}(1)\right) \approx \frac{-1.05449}{-\sqrt{3}} \approx 0.6088
$$

\section{Counting the density of S5}

The case of S5 logic is even more complicated than in Grzegorczyk's one. There is no nice estimation of its density with the one exception of trivial logic(vi) The only thing we can do is counting the floating value of its density. To do this let us consider the following system of equations corresponding to Table 2

$$
\begin{aligned}
f_{1}= & \left(f_{8} f_{1}+f_{3}\left(f_{1}+f_{2}\right)\right) z+z, \\
f_{2}= & \left(f_{8} f_{2}+f_{1}+f_{2}+f_{6}+f_{7}\right) z, \\
f_{3}= & \left(f_{1}\left(f_{2}+f_{3}+f_{4}+f_{7}\right)+f_{5}\left(f_{3}+f_{4}\right)+f_{6}\left(f_{3}+f_{7}\right)+f_{8} f_{3}\right) z, \\
f_{4}= & \left(f_{6}\left(f_{2}+f_{4}\right)+f_{8} f_{4}+f_{3}+f_{4}+f_{5}\right) z, \\
f_{5}= & \left(f_{3}\left(f_{4}+f_{5}\right)+f_{6}\left(f_{1}+f_{5}\right)+f_{7}\left(f_{1}+f_{2}+f_{4}+f_{5}\right)+f_{8} f_{5}\right) z, \\
f_{6}= & \left(f_{3}\left(f_{6}+f_{7}\right)+f_{4}\left(f_{1}+f_{2}+f_{6}+f_{7}\right)+f_{5}\left(f_{1}+f_{5}\right)+f_{8} f_{6}\right) z, \\
f_{7}= & \left(f_{5}\left(f_{2}+f_{7}\right)+f_{8} f_{7}\right) z, \\
f_{8}= & \left(f_{1}\left(f_{1}+f_{5}+f_{6}\right)+f_{2}\left(f_{1}+f_{2}+f_{3}+f_{4}+f_{5}+f_{6}+f_{7}\right)+f_{3}^{2}+\right. \\
& \left.+f_{4}\left(f_{3}+f_{4}+f_{5}\right)+f_{5}^{2}+f_{6}^{2} f_{7}\left(f_{3}+f_{6}+f_{7}\right)+f_{8} f_{F}+f_{8}\right) z,
\end{aligned}
$$

(v) Because $z_{0}=\frac{1}{3}$ is an algebraical singularity, to count the differential we have to substitute: $\sqrt{1-3 z}:=t$.

(vi) The trivial modal logic $\operatorname{Triv}$ is the one in which $\square p=p$. Then in our case $\operatorname{Grz}^{\leq 1}(1)=\operatorname{Triv}(1)$. 
where

$$
\begin{array}{llll}
f_{1}=f_{A_{1}}, & f_{2}=f_{A_{2}}, & f_{3}=f_{A_{3}}, & f_{4}=f_{A_{4}}, \\
f_{5}=f_{A_{5}}, & f_{6}=f_{B_{1}}, & f_{7}=f_{B_{2}}, & f_{8}=f_{T_{S 5}} .
\end{array}
$$

We do not introduce new symbols for the generating functions for classes of formulas from the algebra $\mathbf{S 5}(1)_{\equiv}$, but of course they are different from the ones for $\mathbf{G r z}^{\leq 3}(1)_{\equiv}$. The exception is the main function $f_{F}$. It is still defined by 23 . By making the same approach as for $\mathbf{G r z} \mathbf{z}^{\leq 3}(1)$ we may solve the system of equations 25]-32 numerically. The expansion of the function $f_{8}=f_{T_{S 5}}$ around its smallest singularity $z_{0}=\frac{1}{3}$ is the following:

\section{Lemma 19}

$$
f_{T_{S 5}}(z) \approx 0.38156-1.05320 \sqrt{1-3 z}+O(1-3 z)
$$

Furthermore

Theorem 20 The density of truth of the logic S5(1) is about $60.81 \%$.

Proof. From Lemma 16 and 19 we get:

$$
\mu(\mathbf{S 5}(1)) \approx \frac{-1.05320}{-\sqrt{3}} \approx 0.6081
$$

\section{Conclusions and open problems}

First, let us compare the result from Theorem 18 with the ones for the logics $\mathbf{G r z}{ }^{\leq 2}(1)$ and $\mathbf{G r z} \mathbf{z}^{\leq 1}(1)$. In (11) it is shown that

$$
\begin{aligned}
& \mu\left(\mathbf{G r z}^{\leq 2}(1)\right) \approx 61.27 \% \\
& \mu\left(\mathbf{G r z}^{\leq 1}(1)\right)=\frac{1}{10}(5+\sqrt{5}) \approx 72.36 \%
\end{aligned}
$$

As we see the difference between $\mu\left(\mathbf{G r z} \leq^{\leq 2}(1)\right)$ and $\mu\left(\mathbf{G r z} \leq^{\leq 3}(1)\right)$ is not significant at all. On the percentage basis, more than $99 \%$ of the tautologies of $\mathbf{G r z}^{\leq 3}(1)$ is also the $\mathbf{G r z}^{\leq 2}(1)$ tautologies. It seems to be very probable that for any $n>1$ the density of truth for logic $\mathbf{G r z}^{\leq n}$ (1) is above the $60 \%$. To prove this, however, we have to deal with a quite complicated calculation. In (10) it is shown that for any $n$, the algebras $\mathbf{G r z}{ }^{\leq n}(1) / \equiv$ have exactly $2^{n}$ elements. The appropriate system of equation consists of $2^{n}$ equations written in terms of $2^{n}$ generating functions. There is no obstacle to apply the Drmota-LalleyWoods Theorem 13. Even if we are not able not count the density explicitly, we know that it exists. From the inclusions (1) we conclude that:

$$
\mu(\mathbf{G r z}(1))<\ldots<\mu\left(\mathbf{G r z}^{\leq n}(1)\right)<\mu\left(\mathbf{G r z}^{\leq n-1}(1)\right)<\ldots<60.88 \% .
$$

It would be desirable to prove that:

\section{Conjecture 1}

$$
\mu(\operatorname{Grz}(1))>60 \%
$$

In this place we should also raise the problem of the chosen language. Even if we have considered language without the negation sign, it may be easily defined by $\sim p:=p \rightarrow \square p$. This is possible because in the algebra $\mathbf{G r z}^{\leq n}(1) / \equiv$, the formula $\square p$ plays the role of the zero-element. On the other side, adding the functor of negation to the considered language leads to obtaining a larger number of different classes and much more complicated calculations. In the case of S5(1) logic, enriching the language leads to 'solving' a sixteen-element algebra. This is because the universal model consists of one two-element cluster, one point validating $p$ and one point not validating $p$. Hence, the dual modal algebra consists of $2^{4}$ elements. In the case of extensions of Grzegorczyk's logic, adding negation involves even more increase of the number of classes. For example, the number of classes of $\mathbf{G r z} \mathbf{z}^{\leq 2}(1) / \equiv$ (with negation) is $2^{6}$. For details see (2), p. 272-275. Adding the functor of possibility involves exactly the same complications as adding the negation. 
Let us notice, that there is no difficulty in changing the $\{\rightarrow, \sim, \square\}$ language into $\{\wedge, \sim, \square\}$ language or into $\{\vee, \sim, \square\}$ one.

Let us also notice that from the quantitative point of view, the logics $\mathbf{G r z}{ }^{\leq 3}(1)$ and $\mathbf{S 5}(1)$ do not differ actually. Of course, it would be very interesting to check whether the same situation holds for a larger number of variables. Such investigation is connected with counting the number of elements of the n-generated universal models for $\mathbf{S 5}$ and $\mathbf{G r z} \mathbf{z}^{\leq n}$ logics. In this place we may only notice that the needed numbers, although are finite, are incomparably larger than in the case of the one-generated models considered in this paper.

Problem 1 Estimation of density of truth of $\mathbf{G r z}{ }^{\leq 3}(n)$ and $\mathbf{S 5}(n)$.

\section{References}

[1] P. Blackburn, M. de Rijke, Y. Venema, Modal Logic. Cambridge University Press, 2001.

[2] A. Chagrow, M. Zakharyaschev, Modal Logic, Oxford Logic Guides 35, 1997.

[3] B. Chauvin, P. Flajolet, D. Gardy, B. Gittenberger, And/Or trees revisited, Combinatorics, Probability and Computing, 13(4-5), July-September 2004, pp 475-497.

[4] P. Flajolet and A.M. Odlyzko, Singularity analysis of generating functions, SIAM J. on Discrete Math., 3(2), 1990, pp 216-240.

[5] P. Flajolet, R. Sedgewick, Analitic combinatorics: functional equations, rational and algebraic functions, INRIA, Number 4103, 2001.

[6] D. Gardy and A.R. Woods, And/or tree probabilities of Boolean functions, Discrete Mathematics and Theoretical Computer Science, 2005, pp 139-146.

[7] G.E. Hughes, M.J. Cresswell, An Introduction to Modal Logic, Methuen, London, 1968.

[8] M. Huth, M.Ryan, Logic in Computer Science: Modelling and reasoning about Systems, Cambridge University Press, 2000.

[9] Z. Kostrzycka, On the density of implicational parts of intuitionistic and classical logics, Journal of Applied Non-Classical Logics, Vol. 13, Number 3, 2003, pp 295-325.

[10] Z. Kostrzycka, On formulas with one variable in some fragment of Grzegorczyk's modal logic, Bulletin of the Section of Logic, Vol. 33, Number 2, June 2004, pp 95-106.

[11] Z. Kostrzycka, On the density of truth in Grzegorczyk's modal logic, Bulletin of the Section of Logic, Vol. 33, Number 2, June 2004, pp 107-120 .

[12] Z. Kostrzycka, On asymptotic divergency in equivalential logics, submitted, available at http://www.zkostrz.po.opole.pl/publikacje/newpaper1.pdf

[13] Z. Kostrzycka, M. Zaionc, Statistics of intuitionistic versus classical logics, Studia Logica, Vol. 76, Number 3, 2004, pp 307 - 328.

[14] G. Matecki, Asymptotic density for equivalence, Electronic Notes in Theoretical Computer Science URL, 140, 2005 81-91.

[15] M. Moczurad, J. Tyszkiewicz, M. Zaionc, Statistical properties of simple types, Mathematical Structures in Computer Science, Vol. 10, 2000, pp 575-594.

[16] H.S. Wilf, Generating functionology. Second edition. Academic Press, Boston 1994.

[17] A.R. Woods, Coloring rules for finite trees, and probabilities of monadic second order sentences, Random Structures and Algorithms, 10, 1997, pp 453-485.

[18] M. Zaionc, On the asymptotic density of autologies in logic of implication and negation, Reports on 